\title{
Polarization Analysis and Measurement
}

\author{
Dennis H. Goldstein \\ Air Force Wright Laboratory \\ WL/MNGA \\ 101 West Eglin Boulevard, Suite 280 \\ Eglin Air Force Base, Florida 32542
}

\author{
David B. Chenault \\ Nichols Research Corporation \\ Suite C-1, One 11th Avenue \\ Shalimar, Florida 32579

\section{Russell A. Chipman} \\ University of Alabama in Huntsville \\ Physics Department, Optics Building 318 \\ Huntsville, Alabama 35899
} Polarization of light is an important issue in optical system
design, performance, and analysis. Measurement of polar-
ization has progressed past the calibration of polarization
elements and has become a metrology tool used in a wide
variety of fields. Communications, remote sensing, display
technologies, and optical computing are a few of the active
areas of research that require higher quality polarization
elements and better knowledge of instrumental polarization
effects. Mathematical analysis of polarization in optical
systems, elements, and materials has produced a new under-
standing that leads to more precise control of polarization in
optical systems, new applications that exploit polarization
and a variety of new instruments based on polarization.

The SPIE conference Polarization Analysis and Measurement II that took place in July of 1994 was the latest in a series of conferences on polarization that reported on current developments in analysis of polarization in optical systems, design of polarimeters, applications of polarization measurement, and advances in the mathematical understanding of polarization phenomena.

The 17 papers in this special section describe areas of recent intense interest and development in polarization research. The papers may be conveniently divided into three subgroups: (1) design and measurements of optical components and polarization devices, (2) mathematical analysis and modeling of polarization in materials and optical systems, and (3) new developments in polarimetric instrumentation.

The first group of eight papers describes measurements and design of new polarization elements and optical devices. Interference effects that can occur when photoelastic modulators are used with laser sources are described in the first paper. The author describes the effect and its physical source, and presents corrective steps that can be taken to address this important effect. The second paper presents an analysis of a three-mirror system that can be used to produce retarders for the VUV, a region for which few birefringent materials exist. The third paper describes a novel imaging polarimeter and gives examples of its use. The fourth and fifth papers discuss linear polarizers and birefringent waveplates, respectively. The uniformity and performance of these basic elements is important information for optical engineers who are selecting elements for use in their own systems.

In the sixth paper, a magnetically controllable waveplate using ferrofluids is described that holds potential for fast polarimetric modulation. The seventh paper describes retardance and diattenuation measurement results on a PLZT modulator. These measurements show a surprising dependence of diattenuation on applied voltage. The eighth and last paper in the first group gives results of polarization measurements on a diamond-turned mirror as a function of scattering angle.

Significant research has been carried out on the mathematical constructs for polarization analysis and modeling of optical systems, resulting in better understanding of depolarizing systems, the polarization effects of scattering, and the interpretation of measured Mueller matrix data. In the first paper of this second group, the general theory of unitary matrices is applied to the characterization of depolarizing or scattering systems. Additional information about the optical system in question can be extracted from the eigenvectors of the matrix parameterization of the system. The next paper describes the polarization of scattering and gives modeling and experimental results. A third paper in this second group discusses a unified formalism for polarization optics that is useful in understanding complicated polarization phenomenon in optical media, with particular application to optical fibers and fiber optic systems. The next author describes the latest developments in polarization ray tracing with a comparison of the various polarization formalisms and a descrip- 
tion of image formation and aberrations in optical systems. A final paper in this group discusses the application of a matrix representation of the bidirectional reflectance distribution function to radiative transfer.

In the third and last group of papers, a series of two papers describes the optimum angles for the polarization elements in a common configuration for a Stokes polarimeter, a rotating retarder and a fixed polarizer. The next paper documents an imaging liquid-crystal-based polarimeter that has been designed, assembled, and tested at the National Solar Observatory for measurement of solar vector magnetic fields. The instrument uses ferroelectric liquid crystals for polarimetric modulation, and operates at speeds that minimize atmospheric seeing changes. Tests of the liquid crystals and the assembled instrument are given. A final paper describes an in-line fiber optic polarimeter for analyzing the polarization effects of fiber optic cable, fiber optic couplers, isolators, and amplifiers.

The papers in this special section show the rate of progress in polarization instrumentation and demonstrate the breadth of the fields in optics affected by polarization considerations. The contributing authors continue to make significant contributions to the field and we would like to thank each of them for their efforts. We would also like to thank the referees and the staff at Optical Engineering for their excellent support.

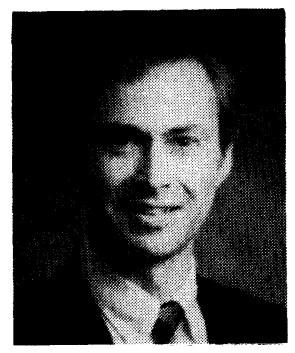

Dennis H. Goldstein obtained his BS and MS from the University of Wisconsin-Madison and his $\mathrm{PhD}$ in physics from the University of Alabama in Huntsville. His research interests include optical processing, polarized light, and electro-optic modulators, and he has five patents in these areas. He currently leads the optical processing activities at the Wright Laboratory Armament Directorate.

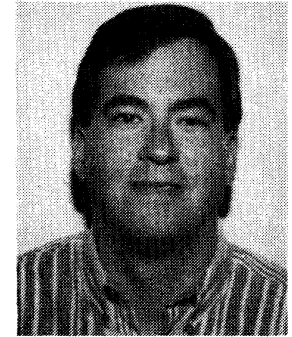

David B. Chenault received his BS in physics from Vanderbilt University in 1986, and his MS and PhD in physics from the University of Alabama in Huntsville in 1989 and 1992, where he developed the infrared spectropolarimeter. He also conducted research in laser polarimetry and high-speed imaging polarimetry during his graduate career. From 1992 to 1994 he was an Office of Naval Technology Postdoctoral Fellow at the Naval Research Laboratory in Washington, D.C., where he developed infrared integrating sphere technology with nonimaging optics. He currently holds a position with Nichols Research Corporation where he is studying infrared phenomenology for infrared scene modeling and analysis.

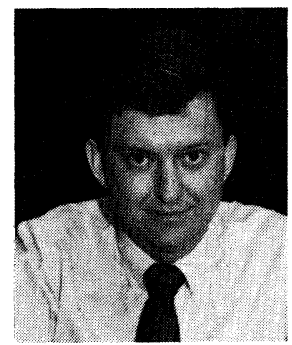

Russell A. Chipman is an associate professor of physics at the University of Alabama in Huntsville, where he heads the Polarization and Lens Design Laboratory. He received his $B S$ in physics from MIT and his MS and PhD in optical science from the University of Arizona. He specializes in polarization issues in optical instrumentation and has pioneered the techniques of polarization ray tracing and of imaging polarimetry for optical system metrology. These techniques extend the methods of conventional optical design and interferometry and provide detailed understanding of the polarization aberrations of polarization critical optical systems, such as optical computers and laser radar systems. His other research interests include optical design, thin films, and microscopy of the eye. He has designed and built several polarimeters for NASA and the Air Force including an infrared spectropolarimeter and several imaging polarimeters. In the 1970s he worked as an optical physicist at Beckman Instruments, Perkin-Elmer, and Space Optics Research Laboratories. He has chaired four SPIE conferences on polarization analysis and polarimetry and taught twelve SPIE short courses on polarization. 